\title{
Compare the Values of Blood Lactate and Heart Rate of Kickboxers during Kickboxing Matches
}

\author{
Mustafa Karadağ \\ Correspondence: Mustafa Karadağ, Firat University, Faculty of Sports Science, 23119, Elazig, Turkey.
}

$\begin{array}{lcc}\text { Received: April 4, } 2017 & \text { Accepted: May 25, } 2017 \quad \text { Online Published: June 1, } 2017 \\ \text { doi:10.11114/jets.v5i7.2317 } & \text { URL: https://doi.org/10.11114/jets.v5i7.2317 }\end{array}$

\begin{abstract}
The aim of this study was to compare the values of heart rate (HR) and blood lactate (BL) of kickboxers during kickboxing match.

This study was conducted with the participation of 18 male and 16 female sportsman in Turkish Kickboxing championship, an organization of Turkish Kickboxing Federation. After the participant athletes read and signed informed voluntary consent form (BGOF) prepared for them, the measurements were collected.

While no significant difference was found between ages, pre-match HR, post-match HR, pre and post-match BL of male and female Kickboxers, a significant difference was statistically found between the height $(\mathrm{P}<0.05)$ and body weights $(\mathrm{P}<0.01)$ of athletes. Age distribution of athletes was determined as $14-23$ and mean age distribution was determined as $17.03 \pm 2.41$. HR pressures of athletes differs according to pre-match and post-match periods $(\mathrm{P}<0.001)$. As $\mathrm{HR}$ frequencies increase by $29.4 \%$ according to pre-match HR regardless of the gender, this increase was determined as $31.4 \%$ for male athletes and $27.2 \%$ for female athletes. While post-match BLs of athletes increase by 4.02 times compared to pre-match regardless of the gender, this increase was determined as 4.20 for male athletes and 3.82 for female athletes.

Kickboxing is a combat sport which has high intensity of anaerobic physical fitness. Matches and rounds for HR and BL concentrations show the importance of the aerobic metabolism in kickboxing. However, the significant rise of HR and BL during simulated match indicates that the anaerobic metabolism is also important in kickboxing.
\end{abstract}

Keywords: kickboxing, blood lactate, heart rate

\section{Introduction}

Combat sports (i.e., taekwondo, karate, judo, kickboxing, muay thai) take an important place in sport world and they require a high level of physical, physiological, complex technical and tactical skills for performance. In generally, these sports have positive effect on physical fitness and general health of athletes (Woodward, 2009; Tabben et al. 2013).

So recent researchers investigated several combat sports practice in among athletes. Most of them reported that combat sports involved a significant development in physical and physiological parameters (Ouergui et al. 2014; Violan et al. 1997; Fukuda et al. 2013). Especially parameters like heart rate (HR) and blood lactate (BL) effect on subsequent performance. It could be explained by the intensity applied during competition. Kickboxing consists of basic skills, combination exercises and sparring drills. It is one of the most popular combine sports in the world has a number of different rulesets. Semi-contact rules or Points Fighting, is an important variant of kickboxing. American kickboxing and/or American full contact karate restricts to strikes using punches and higher kicks; whereas some other arts often regarded as "kickboxing" allow low kicks and even knee strikes, elbows, and grappling maneuvers (Ouergui et al. 2013). It consisted of 3 rounds of 2 minutes each with a rest of 1 minute between rounds (Violan et al. 1997). During matches, kickboxers could perform impact of fighting. This effects to physical and physiological structure of kickboxing athletes. Of course, consists of numerous repetitions of high intensity actions per fight lasting 1-3 s each, separated by low-intensity hopping-stepping movements (Benekeet et al. 2004).

Combat sports involve a significant improvement in physical and motor abilities. That's why kickboxing seems to be not only a practiced for self-defense but also provides general fitness as a contact sport. The aim of our study was to compare the values of $\mathrm{BL}$ and $\mathrm{HR}$ of kickboxers during kickboxing match. 


\section{Material and Method}

\subsection{Subjects}

This study was conducted with the participation of 18 male and 16 female athletes, among athletes, randomly selected based on voluntariness, who participated in national team selections for Turkish Kickboxing Championship organized by Turkish Kickboxing Federation. Means were respectively measured as ( \pm standard deviation) age (male-16.83 \pm 2.43 ; female-17.25 \pm 2.44 ), height (male-174.78 \pm 10.36 ; female-166.75 \pm 6.94 ) and weight (male-68.61 \pm 12.81 ; female-55.81 \pm 6.47 ).

This championship is organized in compliance with the official match procedure of national team selections organization. The participants were informed about the purpose and content of the study and necessary permissions were taken. Also, necessary written and verbal explanations about study and measurements were made and the people who agreed to participate in the study signed "Informed Voluntary Consent Form" (BGOF).

\subsection{Procedures}

To determine the ages of the voluntary participants of the study during the organization, ID card information was taken as basis. The height of the subjects was measured in the standing position by a Stadiometer with a measurement error of $\pm 11 \mathrm{~mm}$. The weight of the subjects was measured by Tanita Digital Scale with a measurement error of $0.5 \mathrm{~kg}$. Body weight was recorded in $\mathrm{kg}$ by measuring with bascule. Body weight index was calculated with the formula body weight $(\mathrm{kg}) /$ body height $\left(\mathrm{m}^{2}\right)$.

Pre-match HR, systolic blood pressure and diastolic blood pressure of participant athletes were measured by using a Microlife BP 3 AS brand device before the match while the athletes were rested and in sitting position. Second and last measurements were collected at the end of 6 minutes match consisting of 3 rounds each of which are 2 minutes long. Between rounds, breaks of 1 minute were given. Athletes in matches resulted in Knock-out or early were not taken into evaluation.

Pre-match HR values of athletes were measured as (male-97.11 \pm 9.80 ; female-99.00 \pm 7.69$)$, post-match HR values as (male-127.56 \pm 11.51 ; female-125.94 \pm 6.90 ), BL pre-match values as (male-4.67 \pm 2.30 ; female-5.04 \pm 2.30 ), BL post-match values as (male-19.63 \pm 4.55 ; female-19.23 \pm 1.74 ). The HR of the subjects was measured using a digital heart monitor (Polar S720i Heart Rate Monitor, Finland). BL level was measured through finger-stick (capillary) blood sampling. The samples were immediately analyzed by Nova Biomedical Blood Analyzer (Kulandaivelan et al. 2009).

\subsection{Statistical Analysis}

The data were analyzed using the SPSS software (IBM SPSS Statistics Version 22). The treatments were compared using student's unpaired $t$-test, and $\mathrm{P}<0.05$ was considered statistically significant. Additionally, HR and BL data were analyzed using the two-way ANOVA and $\mathrm{P}<0.05$ was considered statistically significant.

After receiving a thorough explanation of the protocol, they gave written consent to participate in this study. The study was conducted according to the Declaration of Helsinki and the protocol was fully approved by the Firat University Ethics Committee before the commencement of the assessments.

Table 1. Age, height, body weight, pre-match HR, post-match HR, pre and post-match BL levels of male and female Kickboxers

\begin{tabular}{|c|c|c|c|}
\hline \multirow{2}{*}{ Variables } & \multicolumn{2}{|c|}{ Groups } & \multirow{2}{*}{$\mathbf{P}$} \\
\hline & Male & Female & \\
\hline Age (years) & $16.83 \pm 2.43$ & $17.25 \pm 2.44$ & $N S$ \\
\hline Height $(\mathrm{cm})$ & $174.78 \pm 10.36$ & $166.75 \pm 6.94$ & $P<0,05$ \\
\hline Body Weight (kg) & $68.61 \pm 12.81$ & $55.81 \pm 6.47$ & $P<0,01$ \\
\hline $\operatorname{BMI}\left(\mathrm{kg} / \mathrm{m}^{2}\right)$ & $22,28 \pm 2,78$ & $20,25 \pm 2,20$ & $P<0,01$ \\
\hline Pre-match HR & $97.11 \pm 9.80$ & $99.00 \pm 7.69$ & $N S$ \\
\hline Post-match HR & $127.56 \pm 11.51$ & $125.94 \pm 6.90$ & $N S$ \\
\hline Pre-match BL & $4.67 \pm 2.30$ & $5.04 \pm 2.30$ & $N S$ \\
\hline Post-Match BL & $19.63 \pm 4.55$ & $19.23 \pm 1.74$ & $N S$ \\
\hline
\end{tabular}

Data are presented as means and standard deviations.

NS: Not significant

\section{Finding}

While no significant difference was found between ages, pre-match HR, post-match HR, pre and post-match BL levels of male and female Kickboxers, a significant difference was statistically found between height, body weight and body mass index $(\mathrm{p}<0,05)$. 
HR pressures of athletes differs according to pre-match (resting period) and post-match periods $(\mathrm{P}<0.001)$. While pre-match HR frequencies of all athletes, only male kickboxers and only women kickboxers were respectively 98,97 and $99 \mathrm{~mm} \mathrm{Hg}$, these values respectively increased to 126, 124 and $125 \mathrm{~mm} \mathrm{Hg}$ level (Table 2).

Table 2. Pre and post-match HR value changes of athletes.

\begin{tabular}{lccc}
\hline \multirow{2}{*}{ Variables } & \multicolumn{2}{c}{ HR (mm Hg) } & \multirow{2}{*}{ P } \\
\cline { 2 - 4 } & Pre & Post & $P<0,001$ \\
All athletes & $98.00 \pm 8.79$ & $126.79 \pm 9.52$ & $P<0,001$ \\
Male & $97.11 \pm 9.80$ & $127.56 \pm 11.51$ & $P<0,001$ \\
Female & $99.00 \pm 7.69$ & $125.94 \pm 6.90$ & $P$ \\
\hline
\end{tabular}

Data are presented as means and standard deviations.

As HR frequencies increase by $29.4 \%$ according to pre-match HR regardless of the gender, this increase was determined as $31.4 \%$ and $27.2 \%$ respectively for male and female athletes.

On table 3, pre-match and post-match BL levels of kickboxers are shown. BL levels of kickboxers had increase during post-match periods $(\mathrm{P}<0.001)$. While pre-match $\mathrm{BL}$ levels of all athletes, only male kickboxers and only women kickboxers were respectively 4.84, 4.67 and $5.04 \mathrm{mg} / \mathrm{dL}$, these values respectively increased to $19.44,19.63$ and 19.23 $\mathrm{mg} / \mathrm{dL}$.

While post-match BL levels of athletes increase by 4.02 times compared to pre-match BL level regardless of the gender, this increase was determined as 4.20 and 3.82 for male and female athletes (Figure 3).

Mean height and weight of female kickboxers are respectively $4.6 \%$ and $18.7 \%$ lower than mean height and weight of male kickboxers (Figure 1). Age distribution of athletes was determined as 14-23 and mean age distribution was determined as $17.03 \pm 2.41$.
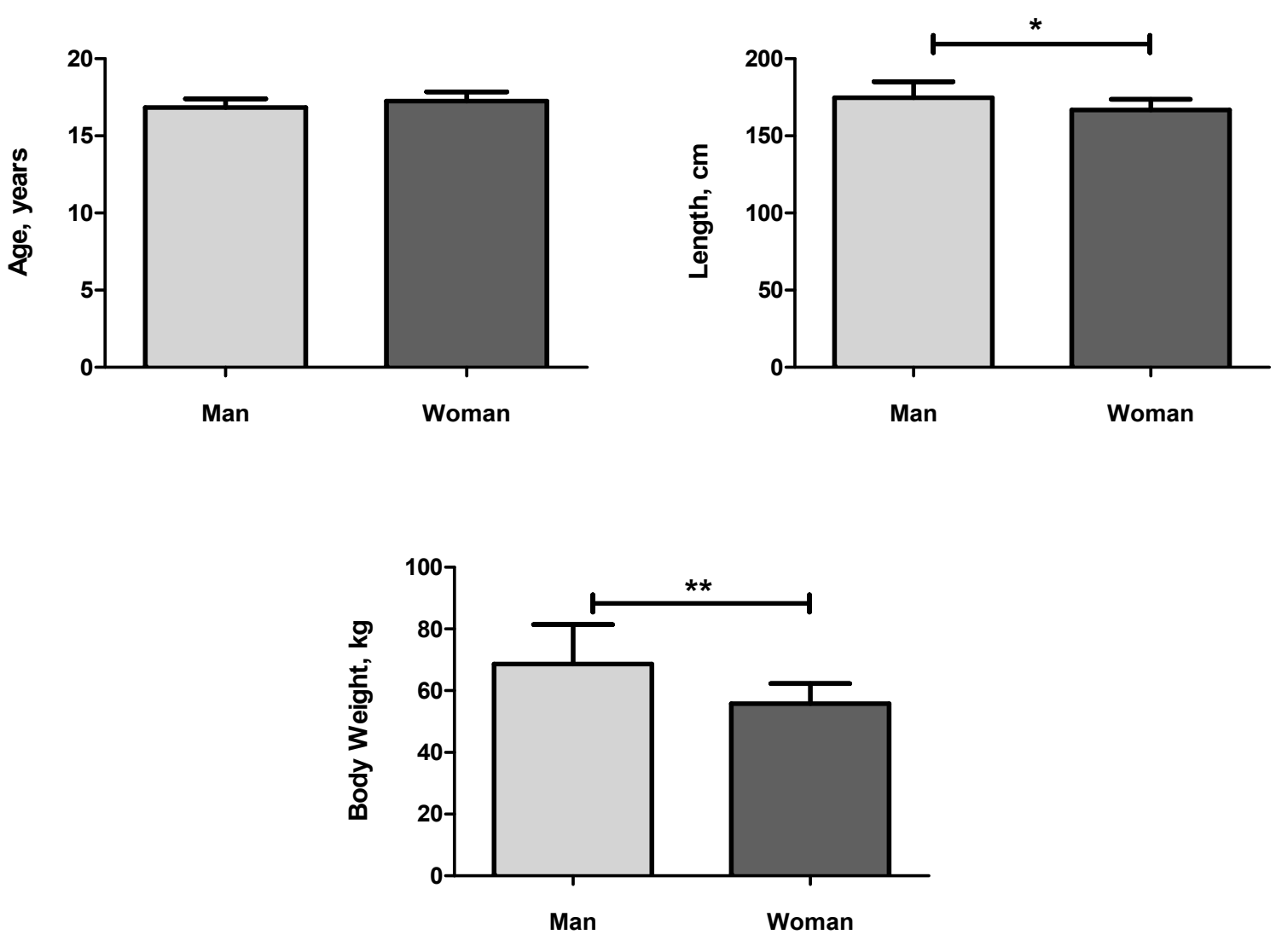

Figure 1. Age, height and body weight distributions according to gender in kickboxers

HR pressures of athletes differs according to pre-match (resting period) and post-match periods $(\mathrm{P}<0.001)$. While pre-match HR frequencies of all athletes, only male kickboxers and only women kickboxers were respectively 98,97 and $99 \mathrm{~mm} \mathrm{Hg}$, these values respectively increased to 127,128 and $126 \mathrm{~mm} \mathrm{Hg}$ level (Table 2). As HR frequencies 
increase by $29.4 \%$ according to pre-match HR regardless of the gender, this increase was determined as $31.4 \%$ and $27.2 \%$ respectively for male and female athletes.

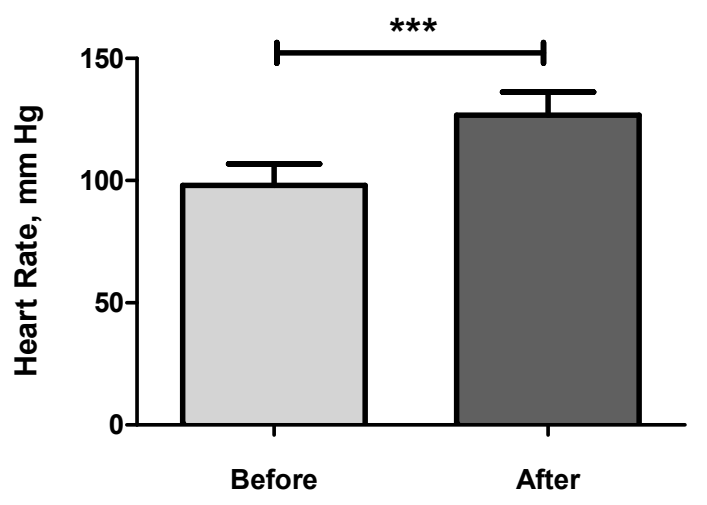

Kick-box athletes

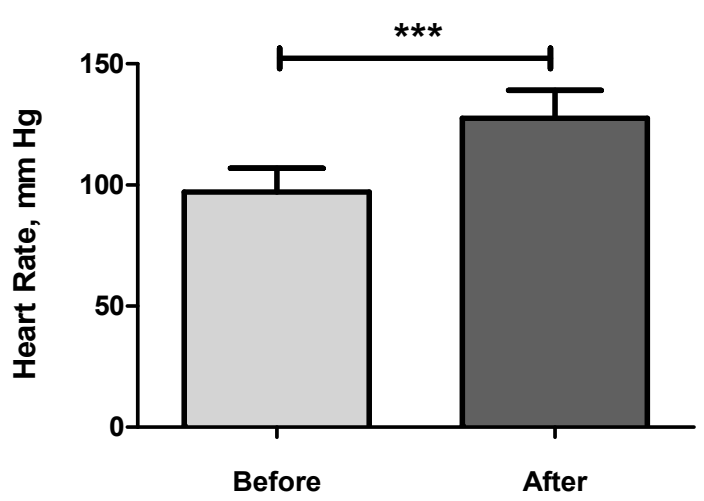

Kick-box athletes only Man

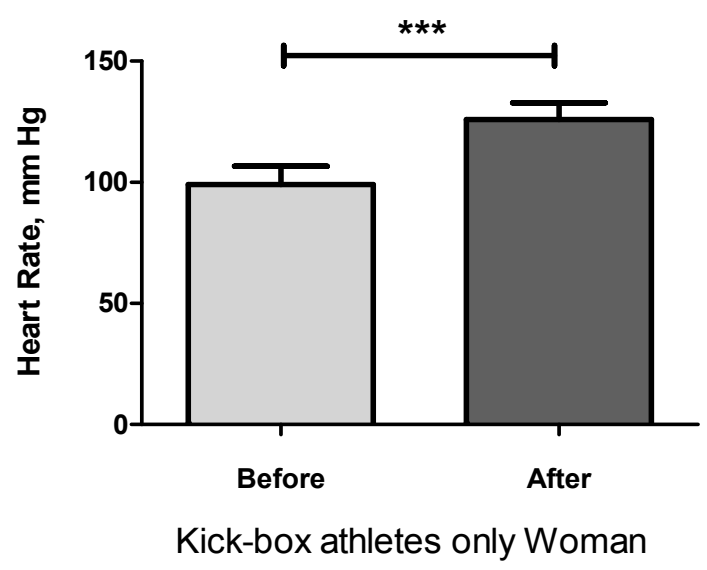

Figure 2. Pre and post training HR changes of kickboxers

BL levels of kickboxers had increase during post-match periods $(\mathrm{P}<0.001)$. While pre-match BL levels of all athletes, only male kickboxers and only women kickboxers were respectively 4.84, 4.67 and $5.04 \mathrm{mg} / \mathrm{dL}$, these values respectively increased to $19.44,19.63$ and $19.23 \mathrm{mg} / \mathrm{dL}$ (Table 3). While post-match BL levels of athletes increase by 4.02 times compared to pre-match BL level regardless of the gender, this increase was determined as 4.20 and 3.82 for male and female athletes (Figure 3). While pre and post-match HR and BL levels of kickboxers are not affected by gender difference $(\mathrm{P}>0.05)$, they showed changes according to pre-training and post-training periods (Table 4; $\mathrm{P}<0.001)$. No interaction was found between gender difference and period (pre and post training) on the HR and BL levels of kickboxers.

Table 3. Pre and post-match BL level changes of athletes

\begin{tabular}{llll}
\hline \multirow{2}{*}{ Variables } & BL $(\mathbf{m g} / \mathbf{d L})$ & & P \\
\cline { 2 - 4 } & Pre & Post & $P<0,001$ \\
All athletes & $4.84 \pm 2.28$ & $19.44 \pm 3.47$ & $P<0,001$ \\
Male & $4.67 \pm 2.30$ & $19.63 \pm 4.55$ & $P<0,001$ \\
Female & $5.04 \pm 2.31$ & $19.23 \pm 1.74$ & \\
\hline
\end{tabular}

Data are presented as means and standard deviations. 


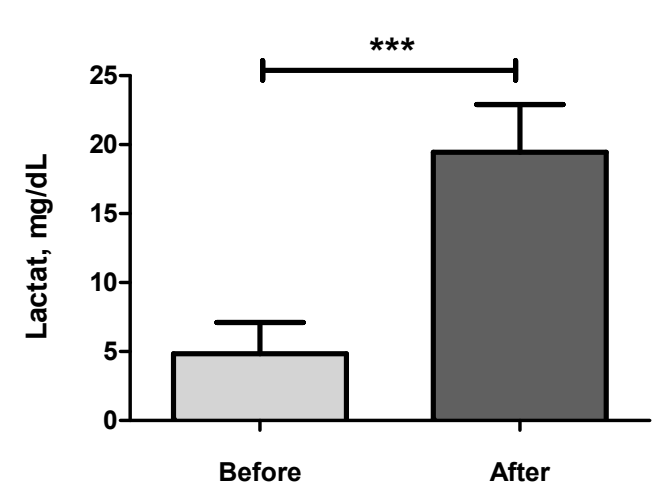

Kick-box athletes

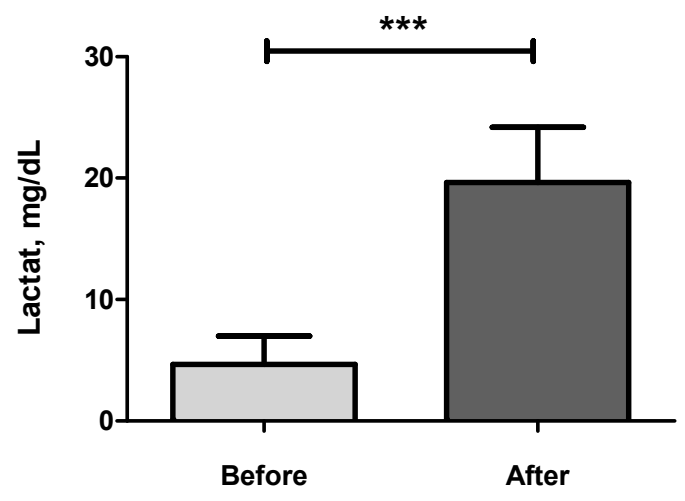

Kick-box athletes only Man

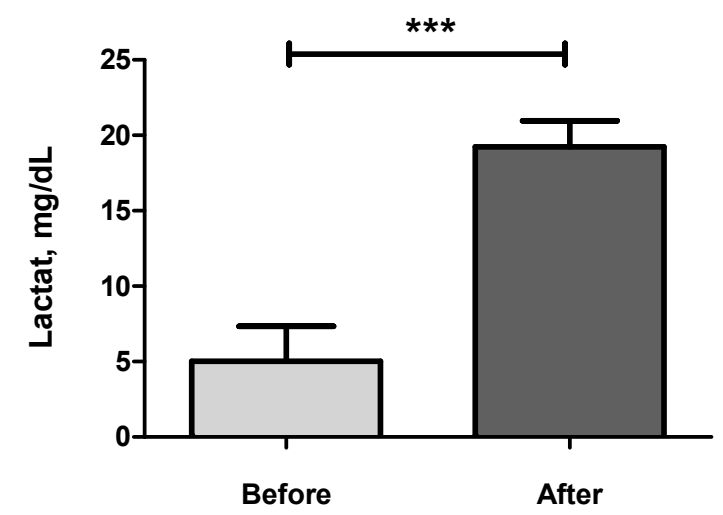

Kick-box athletes only Woman

Figure 3. Pre and post training BL changes of kickboxers
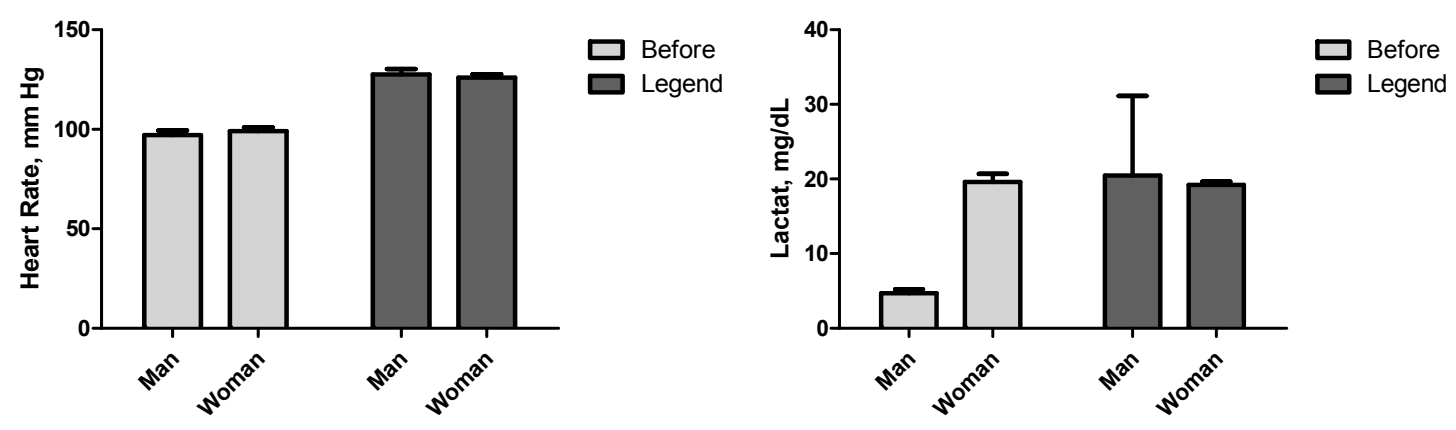

Figure 4. Distribution of pre and post-match HR and BL levels of kickboxers according to gender Table 4. Pre and post-match HR and BL level changes of kickboxers

\begin{tabular}{|c|c|c|c|c|c|c|c|c|}
\hline \multirow{3}{*}{ Variables } & \multicolumn{4}{|c|}{ Groups } & \multirow{3}{*}{ SEM } & \multirow{2}{*}{\multicolumn{3}{|c|}{$P$}} \\
\hline & \multicolumn{2}{|c|}{ Male } & \multicolumn{2}{|c|}{ Female } & & & & \\
\hline & Pre & Post & Pre & Post & & $\mathbf{S}$ & $\mathbf{T}$ & SxT \\
\hline $\mathrm{HR}(\mathrm{mm} \mathrm{Hg})$ & 97.11 & 127.56 & 99.00 & 125.94 & 2.17 & 0.952 & 0.001 & 0.439 \\
\hline $\mathrm{BL}(\mathrm{mg} / \mathrm{dL})$ & 4.67 & 19.63 & 5.04 & 19.23 & 4.12 & 0.983 & 0.001 & 0.600 \\
\hline
\end{tabular}

Two-Way ANOVA, $\mathrm{P}<0.05 \mathrm{~S}=$ Kick-box athletes sex; $\mathrm{T}=$ Time; SxT= Kick-box athletes Sex $\mathrm{x}$ Time interactions 


\section{Discussion}

The literature found that combat sports like taekwondo, kickbox practice rise HRs sufficiently to increase cardiorespiratory fitness. Furthermore, HR response and BL concentrations measured during competition may depend on the level of the opponent, the position of the athlete or the trainer and the impact of the kick and fist. In generally, HR and BL values show the maximal character of the competition. It was imposed the all-round for all subjects. Sometimes cardiovascular demands remained stable through the matches. This result is similar to that reported by another research who observed a small variation in HR in two karate combat simulations, one of 2 min and another of 3 min duration (Bouhlel et al. 2006; Lide et al. 2008).

In our study, while no significant difference was found between ages, pre-match HR, post-match HR, pre and post-match BL levels of male and female Kickboxers, a significant difference was statistically found between the height $(\mathrm{P}<0.05)$ and body weights $(\mathrm{P}<0.01)$ of athletes.

HR values of athletes differs according to pre-match and post-match periods $(\mathrm{P}<0.001)$. While pre-match HR frequencies of all athletes, only male kickboxers and only women kickboxers were respectively 98,97 and $99 \mathrm{~mm} \mathrm{Hg}$, these values respectively increased to 126,124 and $125 \mathrm{~mm} \mathrm{Hg}$ level. As HR frequencies increase by $29.4 \%$ according to pre-match HR regardless of the gender, this increase was determined as $31.4 \%$ and $27.2 \%$ respectively for male and female athletes.

The recovery process in combat sport like kickboxing has required when the athlete may have to compete on more than one occasion during a competition held in a single day (Monedero et al. 2000). It is known that active recovery is its ability to assist lactic acid removal. Otherwise athlete can feel pain, and suffer a decrease in athletic performance. Athlete attainments better performances when it was applied (Bond et al. 1991). Recent studies showed the recovery process after combat simulation. But some studies measured BL concentration after combat sports specific tasks and no performance difference was found (Ibrahim et al. 2014). In a study, HR showed a variation during the whole time of kickboxing match and attained a value of $180 \pm 5 \mathrm{bpm}$ for Active Recovery. Many studies have reported a significant increase in HR during matches of different striking combat sports (Bouhlel et al. 2006; Campos et al. 2012).

In our study, BL levels of kickboxers had increase during post-match periods $(\mathrm{P}<0.001)$. While pre-match BL levels of all athletes, only male kickboxers and only women kickboxers were respectively $4.84,4.67$ and $5.04 \mathrm{mg} / \mathrm{dL}$, these values respectively increased to 19.44, 19.63 and $19.23 \mathrm{mg} / \mathrm{dL}$. While post-match BL levels of athletes increase by 4.02 times compared to pre-match BL level regardless of the gender, this increase was determined as 4.20 and 3.82 for male and female athletes.

Another study showed that BL increased significantly during the combat and reached $14.93 \pm 0.71 \mathrm{mmol} .1-1$ for Active Recovery group and 14.87 \pm 0.69 mmol.1-1 for Passive Recovery group. These results demonstrated an activation of the anaerobic glycolysis during combat. Amtmann et al. have reported a mean value of BL of $15.2 \pm 4$ mmol.l-1 after a match (Amtmann et al. 2008). The kickbox values measured in a study were higher than those measured in other striking combat sports such as boxing, kung fu, muay thai and taekwondo, suggesting that kickboxing seems to rely more on anaerobic glycolysis compared to these other striking combat sports (Ibrahim I, et al. 2014). Touguinha et al. found no difference in BL concentration after 9 min of AR or PR after a judo specific task (Touguinha et al. 2011). During exercise, particularly short-term high intensity exercise, muscles produce lactate rapidly, whereas lactate clearance is slowed. Later during recovery from short-term exercise, there is net lactate uptake from the blood by resting muscles or other muscles that are doing mild to moderate exercise (Gür, 2012).

Also our study was not found a statistical significant between female and male kickboxers compare to BL values of winner and loser $(\mathrm{P}>0.01)$. But the HR and $\mathrm{BL}$ changes in our study prove the high intensity of the match. Especially, during official match and top level trainings athletes could perform several matches in the same day prior to undertaking a final's match. Thus, kickboxers can provide an adaptation to this situation as physiological and psychological quickly.

Within the general approach, it is believed that while metabolic responses seen during endurance exercise cause some adaptations in this kind of exercise, it does not result in any significant difference on strength and power outputs. However, it has been observed that strength trainings aimed to increase muscle mass relatively increase BL level more. None the less, little is known about the metabolic stress on the organism caused by strength/power trainings in practice. Kickboxing features dynamic/power training (explosive and/or ballistic moves are made, low in severance, resting periods are not too long or too short, and content of the training is lower). Age, gender and training states which affect acute lactate production (Atabek, 2009).

In conclusion; kickboxing has high intensity of both aerobic and anaerobic physical fitness. Match and rounds for HR and BL concentrations show the importance of the aerobic metabolism in kickboxing. However, the significant rise of $\mathrm{HR}$ and BL during simulated match indicates that the anaerobic metabolism is also important in kickboxing. Especially, kickboxer trainer should use this results in their trainings. The HR and blood lactate changes prove the high intensity of the competition. 


\section{Acknowledgment}

The study was approved by Firat University Non-Interventional Research Ethics Committee Decision No: 177608, Date: 23 Mar 2016.

\section{References}

Amtmann, J. A., Amtmann, K. A., \& Spath, W. K. (2008). Lactate and rate of perceived exertion responses of athletes training for and competing in a mixed martial arts event. J. Strength Cond. Res., 22, 645-647. https://doi.org/10.1519/JSC.0b013e318166018e

Atabek, H. Ç. (2009). Acute Lactate Production during Resistance Training. SPORMETRE Journal of Physical Education and Sport Science, VII(1), 29-36.

Beneke, R., Beyer, T., Jachner, C., et al. (2004). Energetics of karate kumite. Eur. J. Appl. Physiol., 92, 518-523. https://doi.org/10.1007/s00421-004-1073-x

Bond, V., Adams, R. G., Tearney, R. J., et al. (1991). Effects of active and passive recovery on lactate removal and subsequent isokinetic muscle function. J. Sports Med. Phys. Fitness, 31, 357-61.

Bouhlel, E., Jouini, A., Gmada, N., et al. (2006). Heart rate and blood lactate responses during Taekwondo training and competition. Sci. Sports, 21, 285-90. https://doi.org/10.1016/j.scispo.2006.08.003

Campos, F. A. D., Bertuzzi, R., Dourad, A. C., et al.(2012). Energy demands in taekwondo athletes during combat simulation. Eur. J. Appl. Physiol., 112, 1221-8. https://doi.org/10.1007/s00421-011-2071-4

Fukuda, D. H., Stout, J. R., Kendall, K. L., Smith, A. E., Wray, M. E., \& Hetrick, R. P. (2013). The effects of tournament preparation on anthropometric and sport-specific performance measures in youth judo athletes. $J$. Strength Cond. Res. Natl. Strength Cond. Assoc., 27(2), 331-339. https://doi.org/10.1519/JSC.0b013e31825423b3

Gür, E. (2012). A Comparison of Blood Lactate Level and Heart Rate Following a Peak Anaerobic Power Test in Different Exercise Loads. European Journal of Experimental Biology, 2(5), 1854-1861.

Ibrahim, I. et al. (2014). Effects of Recovery Type after a Kickboxing Match on Blood Lactate and Performance in Anaerobic Tests. Asian J. Sports Med., 5(2).

Kulandaivelan, S., Verma, S. K., Mukhopadhyay, S., \& Vignesh, N. J. (2009). Test Retest Reproducibility of a Hand-Held Lactate Analyzer in Healthy Men. Journal of Exercise Science and Physiotherapy, 5(1), 30-33.

Lide, K., Imamura, H., Yoshimura, Y., et al. (2008). Physiological responses of simulated karate sparring matches in young men and boys. J. Strength Cond. Res., 22, 839-844. https://doi.org/10.1519/JSC.0b013e31816a5af6

Monedero, J. (2000). Donne B. Effect of recovery interventions on lactate removal and subsequent performance. Int. J. Sports Med., 21, 593-597. https://doi.org/10.1055/s-2000-8488

Ouergui, I., Hssin, N., Franchini, E., Gmada, N., \& Bouhlel, E. (2013). Technical and tactical analysis of high level kickboxing matches. Int. J. Perform Anal. Sport, 13(2), 294-309).

Ouergui, I., Hssin, N., Haddad, M., Padulo, J., et al. (2014). The effects of five weeks of kickboxing training on physical fitness. Muscles, Ligaments and Tendons Journal, 4(2), 106-113.

https://doi.org/10.11138/mltj/2014.4.2.106

Tabben, M., Sioud R., Haddad, M., et al. (2013). Physiological and Perceived Exertion Responses during International Karate Kumite Competition. Asian J. Sports. Med., 4(4), Dec. https://doi.org/10.5812/asjsm.34246

Touguinha, H. M., Silva, F. F., Carvalho, W., et al. (2011). Effects of active vs. passive recovery on blood lactate after specific judo-task. JEP online, 14, 54-61.

Violan, M. A., Small, E. W., Zetaruk, M. N., \& Micheli, L. J. (1997). The effect of karate training on flexibility, muscle strength, and balance in 8- to 13-year-old boys. Pediatr. Exerc. Sci., 9(1), 55-64. https://doi.org/10.1123/pes.9.1.55

Woodward, T. W. (2008). A review of the effects of martial arts practice on health. Wis. Med. J. WMJ., 108(1), 40.

\section{Copyrights}

Copyright for this article is retained by the author(s), with first publication rights granted to the journal.

This is an open-access article distributed under the terms and conditions of the Creative Commons Attribution license which permits unrestricted use, distribution, and reproduction in any medium, provided the original work is properly cited. 